\title{
Management Strategies for a Defined Contribution Pension Fund under the Hull-White Interest Rate Model
}

\author{
Patrick Kandege Mwanakatwe ${ }^{*}$, Lixin Song and Emmanuel Hagenimana \\ School of Mathematical Sciences, Dalian University of Technology, Dalian, China \\ ${ }^{*}$ Corresponding author
}

\begin{abstract}
This paper analyzes optimal investment strategies for a DC pension fund under the Hull-White interest rate model. Under this model, the pension fund manager can invest capital in the bank account, stock index, and real estates. The dynamics of the interest rate follows the Hull-White interest rate model, and a drifted Brownian motion drives the financial market. The pension fund manager aims to maximize the expected terminal utility of wealth in a complete market setting under constant relative risk aversion (CRRA). This paper derives the Hamilton-JacobiBellman (HJB) equation associated with the control problem using a dynamic programming technique. We obtain the explicit solution for optimal investment policies by solving the HJB equation and optimal value function. The results show that the optimal proportion invested in risky assets is higher in stock than in a real estate.
\end{abstract}

Keywords-optimal investment strategy; defined contribution pension fund; stochastic dynamic programming; interest rate

\section{INTRODUCTION}

The fundamental idea of the pension fund is to finance the benefits to the particular group of recipients after retirement. These benefits are paid over a fixed period or for life. Managing such funds requires continuously monitoring the risk exposure and regularly rebalancing assets.[1] reported that the benefits are the results of three combined processes namely; accumulation of contributions, the investment returns of contributions and the presence of minimum guarantees on the benefits at retirement (guarantee on the lump sum) or after retirement (guarantee on annuities).

Today, studies on the management of DC pension fund in the presence of stochastic interest rate has gained remarkable considerations in actuarial science and financial mathematics. For example, [2-5] presented comprehensive materials and theories of DC pension in the stochastic interest rate. In these studies, the interest rate is considered as a Vasicek type and taking the affine structure. Also, the affine structure has been studied extensively in the following works [6-10].

The stochastic dynamic programming method for solving DC pension fund problems is reported in [2], [6] [11], [12] and the reference therein. These studies aimed to maximize the utility of terminal value of DC pension fund during the accumulation phase. In these studies, martingale and duality approach was applied to find exact solutions for the CRRA utility function. Similarly, [4] used the stochastic dynamic programming to solve the continuous-time optimal portfolio management problem of DC pension plans. Under this study, the contribution flows, interest rates, and inflation rates are assumed to follow the stochastic model. Also, [7] used stochastic dynamic programming method to examine the optimal asset allocation for a DC pension plan with downside protection under stochastic inflation.

Likewise, [13] discussed the portfolio optimization problem for an investor seeking to find the maximum expected utility of the terminal capital in a DC pension plan. The approach focused on a constant elasticity of variance (CEV) model, which describe the stock price dynamics without considering minimum guarantee on the terminal wealth. The exact solutions for the CRRA and CARA (Constant absolute risk aversion) utility function determined using stochastic optimal control, variable change technique, and power transform. In the same line, [5] applied optimal stochastic control method to investigate optimal asset allocation for aggregated defined benefits pension fund with the stochastic interest rate. The study aimed to minimize deviations of the unfunded actuarial liability from zero along a finite time horizon.

Moreover, [9] applied the HJB equation, Legendre transformation, and dual theory to find the optimal investment strategies of DC pension fund. The proposed models included the stochastic interest rate. They assumed that the pension fund manager could invest in a risk-free asset, a zero-coupon bond, and a single risky asset.

Comprehensive reviews concerning the optimal asset allocation and the dynamics of interest rate are modeled by; Vasicek model, Cox-Ingersoll-Ross (CIR) model and affine interest rate models (this combine both Vasicek and CIR). Although these approaches are used in practice, Vasicek model provides unique characteristics, in which the interest rate can be treated using normally distributed technique and simplifies the optimal portfolio strategy [2], [4], [5], [14].

For decades, various researchers have used Vasicek model, CIR model and affine models to study investment strategies and optimal management of a DC pension under the stochastic framework. However, these models are built upon a particular diffusion process with constant (i.e., time-independent) coefficients, which is not suitable for solving problems related to DC pension fund. So, the investment decision for a pension plan should not be treated as a constant value because the interest 
rate is stochastic taking an extended period. Therefore, it is suitable to consider the future interest rate as the time-dependent.

In this view, the present study attempts to find the optimal investment strategy for DC pension fund in an uncertain environment whose interest rate follows the Hull-White model. The risky assets price and the contributions are modeled using the geometric $\checkmark$ motion, and the optimal investment strategies are derived using CRRA utility function. The most important feature of the Hull-White model as revealed in [15] it provides complete structure and the term structure of forward rate volatilities. The main novelty of this paper is in the following; it proposes the use of the Hull-White interest model to describe the behavior of optimal investment strategies for a DC pension fund; find the explicit solution for optimal investment strategies, we give simulation results and explain the impact of model parameters on the investment strategies.

This paper is organized as follows: Section II presents the mathematical model. Section III gives the proposed optimization problem and the explicit solution for CRRA utility function. In section IV the numerical example is presented to illustrate the efficiency of the proposed model. Finally, some concluding remarks are given in section 5.

\section{MATHEMATICAL MODEL}

In this section, we present the market structure and describe the stochastic dynamics of the asset values and the contributions. We consider a frictionless and complete financial market, which is continuously open over the fixed time interval $[0, T]$, where $T>0$ stands for the retirement time. Let $\left(\Omega, \mathcal{F}, \mathcal{F}(t)_{t \geq 0}, \mathrm{P}\right)$ be a filtered complete probability space and $\mathcal{F}(t)$ represents the information available before time $t$ in the market. We assume that all the processes introduced below are well defined and adapted from $\mathcal{F}(t)_{t \geq 0}$.

\section{A. The Financial Market}

The financial market consists of three classes of assets namely fixed income securities (Bond/Bank account), equities (stocks) and real estate securities (property). Bond/bank account is considered as risk-free assets while stock and real estate are in risky assets categories. These categories of assets operate independently and possess different risks depending on market conditions.

1) Fixed income securities: The first asset in a financial market is the risk-free asset (bank account or bond) denoted by $B(t)$ and the price of this asset at time $t$ evolve according to the differential equation:

$$
d B(t)=B(t) r(t) d t, B(0)=B_{0}, r(t)>0,
$$

where $B_{0}$ is the initial price of the risk-free asset, and $r(t)$ is the instantaneous rate of interest. We assume that the instantaneous risk-free interest rate $r(t)$ follows Hull-White model (1990). Based on the historical probability measure $P$, the dynamics of $r(t)$ is given by the mean-reverting stochastic differential equation (SDE) as:

$$
d r(t)=(\theta(t)-\beta r(t)) d t+\sigma d W(t), r(0)=r_{0},
$$

where $\sigma>0, \theta(t)$, and $\beta$ denote; the interest rate volatility, the mean reversion or long-run mean which is time-dependent, and the reversion rate (speed) respectively. $W(t)$ is a standard Brownian motion.

2) The stock: The second asset in the financial market is stock denoted by $S(t)$ whose dynamic follows stochastic differential equation given by:

$$
d S(t)=S(t) \theta_{S}(t) d t+\sigma_{S} d W_{S}(t), S(0)=S_{0}
$$

where $S_{0}, \theta_{S}(t)$ and $\sigma_{s}$ denote; the initial stock price, the expected instantaneous rate of return, and the stock volatility respectively.

3) The real estate security: The third asset in the financial market is a real estate; this is a significant investment asset and a potential contributor to pension fund wealth. In this study, we denote property by $R(t)$, with the assumption that dynamics follows stochastic differential equation expressed as:

$$
d R(t)=R(t) \theta_{R}(t) d t+\sigma_{R} d W_{R}(t), R(0)=R_{0},
$$

where $R(0)>0, \theta_{R}(t)$, and $\sigma_{s}>0$ are the initial returns, expected rate of return, and the real estate price volatility respectively.

\section{B. Contributions}

In the defined contribution management, the fund members will be continuously contributing the part of their salaries to retirement time $T$. A completeness of a market takes the instantaneous contributions, denoted by $C(t)$. The dynamics of contributions process at time $t$ is given by the following stochastic differential equation:

$$
d C(t)=C(t) \theta_{C}(t) d t+\sigma_{C} d W_{C}(t), C(0)=C_{0},
$$

where $\theta_{C}>0, \sigma_{C}>0$, and $C_{0}>0$ are; the expected rate of contributions, the contributions volatility, and the initial contribution respectively.

\section{The Wealth Process and Management}

In this study, the proportion of fund invested in the risk-free assets is given by $u_{B}(t)=1-u_{B}(t)-u_{R}(t)$, where $u_{B}(t)$ and $u_{R}(t)$ are proportions of the pension fund invested in the stock and the real estates respectively.

Lemma 2.1: If $X(t)$ is the wealth of a pension fund at time $t \in[0, T]$, and $\left\{\left(u_{S}(t), u_{R}(t)\right): t \geq 0\right\}$ is a Markovian control process adapted to filtration $\mathcal{F}(t){ }_{t \geq 0}$ satisfying the condition;

$$
\mathrm{E} \int_{0}^{T}\left(u_{S}^{2}(t)+u_{R}^{2}(t)\right) d t<\infty
$$

Then, the dynamics of fund under the investment strategy $\left(u_{S}(t), u_{R}(t)\right)$ is given by:

$$
d X(t)=X(t)\left[\begin{array}{c}
\left.u_{B}(t) \frac{d B(t)}{B(t)}+u_{S}(t) \frac{d S(t)}{S(t)}+u_{R}(t) \frac{d R(t)}{R(t)}\right]+ \\
C(t) d t
\end{array}\right.
$$


where $X(0)=X_{0}$ is the initial wealth. By substituting equations (1), (3) and (4) in equation (7), the evolution of the wealth can be expressed as:

$$
\begin{gathered}
d X(t)=X(t)\left[r(t)+u_{S}(t) \sigma_{S} \lambda_{S}+u_{R}(t) \sigma_{R} \lambda_{R}\right] d t+C(t) d t+ \\
X(t)\left[u_{S}(t) \sigma_{S} d W_{S}(t)+u_{R}(t) \sigma_{R} d W_{R}(t)\right], X(0)=X_{0} .
\end{gathered}
$$

\section{III.THE OPTIMIZATION PROGRAM}

The primary aim of the fund manager is to select a portfolio strategy to maximize the expected value of a terminal wealth. We assume that a plan member will retire at time $T$ and is risk averse. The utility function is denoted by $U(x)$, and the optimal control problem by:

$$
\max _{u(t)} E(U(X(T))) \text { s.t (2) and (8) }
$$

whereas $\left(u_{S}(t), u_{R}(t)\right) \in u(t)$.

\section{A. The Value Function and the Hamilton-Jacob-Bellman (HJB) Equation}

In stochastic dynamic programming, we define the value function of the problem as the conditional expectation. It follows from equation (9), the value function of optimal control problem for a strategy $u(t)$ is given by:

$$
\left.W(t, r, X)=\max _{u(t)} E(U(X(T))) \mid X(t)=x, r(t)=r\right) .
$$

The maximum principle leads to the following HJB equation:

$$
\begin{aligned}
W_{t}+ & (\theta(t)-\beta r) W_{r}+\frac{1}{2} X^{2} W_{x x}\left[u_{S}^{2}(t) \sigma_{S}^{2}+u_{R}^{2}(t) \sigma_{R}^{2}\right] \\
+ & \frac{1}{2} \sigma^{2} W_{r r}+\left[r X+\left(u_{S}(t) \sigma_{S} \lambda_{S}+u_{R}(t) \sigma_{R} \lambda_{R}\right) X+C\right] W_{x} \\
& +X W_{x r}\left[u_{S}(t) \sigma_{S}+u_{R}(t) \sigma_{R}\right] \sigma=0,
\end{aligned}
$$

where $W_{t}, W_{x}, W_{r}, W_{x r}, W_{x x}$ and $W_{r r}$ denote partial derivatives of first and second order with respect to time, wealth and interest rate respectively. The equation (11) is redefined as:

$$
0=\max _{u} v(u)
$$

At the optimal control $u^{*}$, there are two conditions as:

$$
v\left(u^{*}\right)=0 \text {, and } \frac{\partial v\left(u^{*}\right)}{\partial u}=0
$$

Applying the second order condition in equation (11) we obtain the optimal investment strategies as:

$$
\begin{array}{r}
u_{S}^{*}(t)=-\frac{\lambda_{S} W_{x}}{X \sigma_{S} W_{x x}}-\frac{\sigma W_{x r}}{X \sigma_{S} W_{x x}}, \text { and } \\
u_{R}^{*}(t)=-\frac{\lambda_{R} W_{x}}{X \sigma_{R} W_{x x}}-\frac{\sigma W_{x r}}{X \sigma_{R} W_{x x}}
\end{array}
$$

Substituting equation (13) into equation (11), we obtain the non-linear second order partial differential equation (PDE) for the value function as:

$$
W_{t}+(\theta(t)-\beta r) W_{r}+\frac{1}{2} \sigma^{2} W_{r r}+(r X+C) W_{x}-
$$

$$
\frac{1}{2} \frac{\left(\lambda_{R}^{2}+\lambda_{S}^{2}\right) W_{x}^{2}}{W_{x x}}-\frac{\sigma\left(\lambda_{R}+\lambda_{S}\right) W_{x} W_{x r}}{W_{x x}}-\frac{\sigma^{2} W_{x r}^{2}}{W_{x x}}=0
$$

From equation (14), the stochastic control problem has been transformed into a nonlinear second order PDE which cannot be solved easily using the typical differential techniques. The standard approach to address this kind of PDE is by separation condition. The subsequent section provides the explicit solution.

\section{B. Explicit Solution for the Utility Function}

In this section, we find the explicit solution for the value function and optimal investment strategies by using stochastic optimal control methods. Considering the case of the CRRA utility, and define the utility function $U:\left(x_{0},+\infty\right) \rightarrow \mathbb{R}$, as an increasing, strictly concave, continuously differentiable and satisfies the condition that: $\lim _{x \rightarrow+\infty} U^{\prime}(x)=0, \lim _{x \rightarrow x_{0}} U^{\prime}(x)+\infty$, where $x_{0} \in \mathbb{R} \cup\{-\infty\}$. When these conditions are met, the CRRA utility function is defined as:

$$
U(x)=\frac{X^{\gamma}}{\gamma} \text { s.t } \gamma \in(-\infty, 0) \cup(0,1) \backslash\{0\}, \forall x \geq 0,
$$

where $\gamma$ is the relative risk aversion. Then, we conjecture the solution to equation (14) as:

$$
W(t, r, X)=U(x) f(t, r)
$$

with boundary condition $f(T, r)=1$. Substituting partial derivatives of equation (16) into (14) and the simplification yields the following equation:

$$
\begin{array}{r}
(\gamma-1)\left[f f_{t}+(\theta(t)-\beta r) f f_{r}+\frac{1}{2} \sigma^{2} f f_{r r}\right]+\gamma(\gamma-1)\left(r+\frac{c}{x}\right) f^{2}- \\
\frac{1}{2} \gamma\left(\lambda_{S}^{2}+\lambda_{R}^{2}\right) f^{2}-\gamma \sigma\left(\lambda_{S}+\lambda_{R}^{2}\right) f f_{r}-\gamma \sigma^{2} f_{r}{ }^{2}= \\
0 .
\end{array}
$$

Again, we conjecture a solution to equation (17) in the form:

$$
f(t, r)=A(t) \exp (B(t) r)
$$

with boundary conditions $A(T)=1$, and $B(T)=0$. Substituting partial derivatives of equation (18) into (17) and simplifying we get:

$(\gamma-1)\left[A(t) r B^{\prime}(t)+A^{\prime}(t) B(t)+\frac{1}{2} A(t) B^{2}(t) \sigma^{\wedge} 2\right]+(\gamma-$

1) $\left(r+\frac{C}{x} A(t)-\frac{1}{2} \gamma\left(\lambda_{S}^{2}+\lambda_{R}^{2}\right) A(t)-\gamma \sigma\left(\lambda_{S}+\lambda_{R}\right) A(t) B(t)-\right.$

$\lambda \sigma^{2} A(t) B^{2}(t)=0$.

Furthermore, removing the dependency on $r$ in equation (19) results to ODEs:

$(\gamma-1) A^{\prime}(t)+M(t) A(t)=0, B^{\prime}(t)-\beta B(t)+\gamma=0$.

For simplicity, we denote $M(t)$ by:

$$
\begin{gathered}
M(t)=(\gamma-1)\left(B(t) \theta(t)+\frac{1}{2} B^{2}(t) \sigma^{2}+\gamma \frac{C}{x}\right)-\frac{1}{2} \gamma\left(\lambda_{S}^{2}+\lambda_{R}^{2}\right)- \\
\gamma \sigma\left(\lambda_{S}+\lambda_{R}\right) B(t)-\gamma \sigma^{2} B^{2}(t)=0 .
\end{gathered}
$$


Solving ODEs in equation (20) with boundary conditions $A(T)=1$ and $B(T)=0$ we obtain the values of $A(t)$ and $B(t)$ as:

$$
\begin{aligned}
& A(t)=\exp \left\{\frac{1}{(1-\gamma)}(M(t)-M(T))\right\}, \text { and } \\
& B(t)=\frac{\gamma}{\beta}\left(1-e^{-\beta(T-t)}\right)
\end{aligned}
$$

Therefore, we summarize the solution by the theorem below.

Theorem 3.1: The optimal investment strategies for a DC pension fund and the value function with power utility function under the Hull-White stochastic interest rate model are given by equations (23)-(25) as:

$$
\begin{gathered}
u_{S}^{*}=\frac{(\theta(t)-r(t))}{(1-\gamma) \sigma_{S}^{2}}+\frac{\sigma B(t)}{(1-\gamma) \sigma_{S}}, \\
u_{R}^{*}=\frac{(\theta(t)-r(t))}{(1-\gamma) \sigma_{R}^{2}}+\frac{\sigma B(t)}{(1-\gamma) \sigma_{R}}, \quad \text { and } \\
W(t, r, X)=\frac{X^{\gamma}}{\gamma} A(t) \exp (B(t) r(t)) .
\end{gathered}
$$

Whereby equation (23) and (24) denote the optimal investment strategies in stock and real estate respectively. Equation (25) is for the value function. In addition to that, the values of $A(t)$ and $B(t)$ are given by equation (22).

The above theorem permits to review optimal investment strategies expressions which are formed by two terms. The first term of the optimal policy gives the effects of risk aversion, instantaneous volatility, and the risk premium on the investment strategies. The second term presents the effects of interest rate volatility, risk aversion, risky asset volatility and the reversion rate on investment strategy. This term reflects the pension manager's decision to hedge the volatility risk.

\section{NUMERICAL ILLUSTRATION}

A numerical example of the proposed model was given to demonstrate the dynamic behavior of a DC pension fund and optimal investment strategy. Tanzania-National Social Security Fund (NSSF) real data was used to illustrate the efficiency of the proposed model. The parameters used are summarized in Table I below.
TABLE I. PARAMETERS AND THEIR RESPECTIVE VALUE

\begin{tabular}{|l|c|c|}
\hline Name of a parameter & Symbol used & Numerical value \\
\hline Initial interest rate & $r_{0}$ & 0.065 \\
Interest rate volatility & $\sigma$ & 0.016 \\
Reversion rate & $\beta$ & 0.4 \\
Mean reversion & $\theta(t)$ & 0.067 \\
\hline Initial stock value & $S_{0}$ & 0.10 \\
Stock means return & $\theta_{S}(t)$ & 0.116 \\
Stock volatility & $\sigma_{S}$ & 0.095 \\
Initial Real estate value & $R_{0}$ & 0.029 \\
Real estate mean return & $\theta_{R}(t)$ & 0.035 \\
Real estate volatility & $\sigma_{R}$ & 0.01 \\
\hline Initial contribution & $C_{0}$ & 0.026 \\
Mean contribution & $\theta_{C}(t)$ & 0.1 \\
Contribution volatility & $\sigma_{C}$ & 0.064 \\
Risk aversion degree & $\gamma$ & 0.5 \\
Time horizon (years) & $T$ & 10 \\
\hline
\end{tabular}

\section{A. Results}

Figure 1 describes the evolution of the stock and real estate prices over time under the Hull-White interest model. It shows that the stock price increases over time with a very wild fluctuation of price, while the price growth for the real estate asset seems to be steady over time.

Figure 2 shows the influence of time on the optimal investment strategies. It reveals that the optimal investment policies increase with time. That is, as the time passes on, investment in riskless asset decreases. Results suggest that the pension fund manager maintains diversifying the portfolio by investing more in stock since the optimal investment strategies in risky assets increase with time.

Figure 3 shows the effect of interest rate volatility $\sigma$ on optimal investment strategies. It shows that increase in the interest rate volatility decreases the optimal investment strategy, this tells the pension fund manager to invest more in a riskless asset to hedge risk. It implies that investment in the risky assets is not suitable when the interest rate volatility increases and viceversa.

Figure 4 presents the impact of mean-reversion rate $\beta$ on the optimal investment strategies. It points out that as the meanreversion rate increases the optimal investment strategies decreases. Further, it depicts that as the time $t$ approaches the maturity time $T$, the risk assets seem to be relatively insensitive to the mean-reversion rate. In practice, to monitor the fluctuations in the interest rate, the risk assets may partially be used to hedge the real interest rate uncertainty.

Figure 5 presents the influence of interest rate on optimal investment strategies. It shows that as interest rate increases, investment in risky assets is not suitable. Therefore, the pension fund managers are advised to increase investment in the riskless asset to hedge risk.

Figure 6 presents the effect varying the degree of riskaversion $\gamma$. The optimal investment strategy is sensitive to the level of risk aversion. Results provide that for a given time horizon the proportion invested in risk assets increase with risk aversion. Unsurprisingly, the higher risk assets yield high returns. 


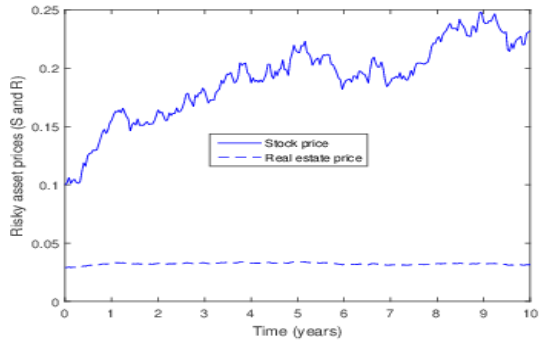

FIGURE I. EVOLUTION OF PRICE OVERTIME

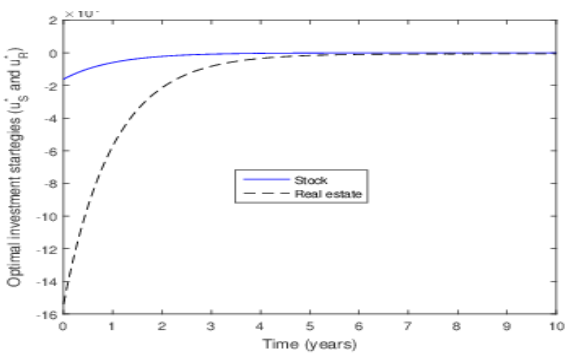

FIGURE II. OPTIMAL INVESTMENT STRATEGIES OVERTIME

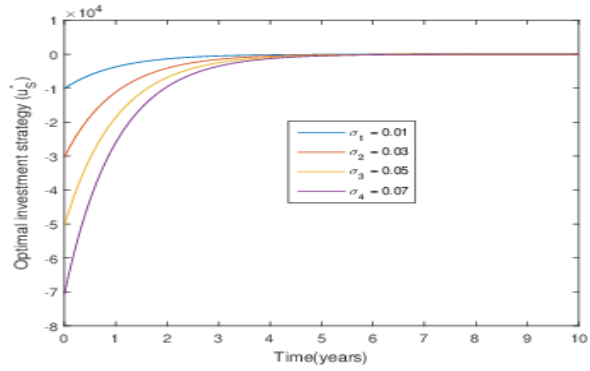

FIGURE III. INFLUENCE OF VOLATILITY $\sigma$ ON STOCK

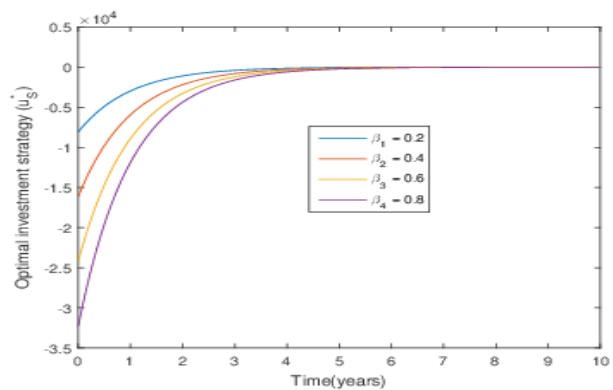

FIGURE IV. EFFECT OF MEAN REVERSION $\beta$ ON STOCK

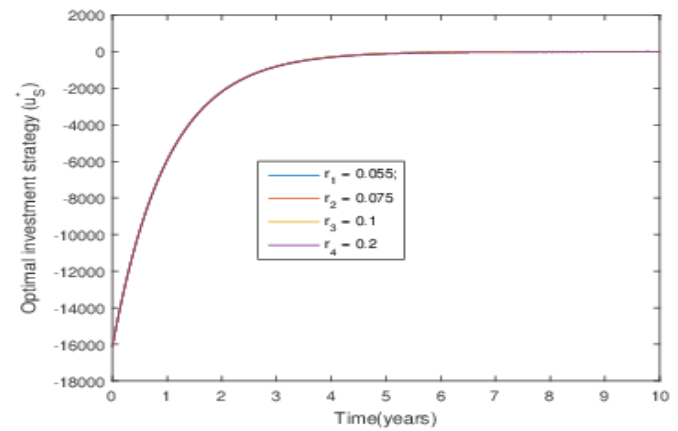

FIGURE V. IMPACT OF INTEREST RATE $r$ ON STOCK

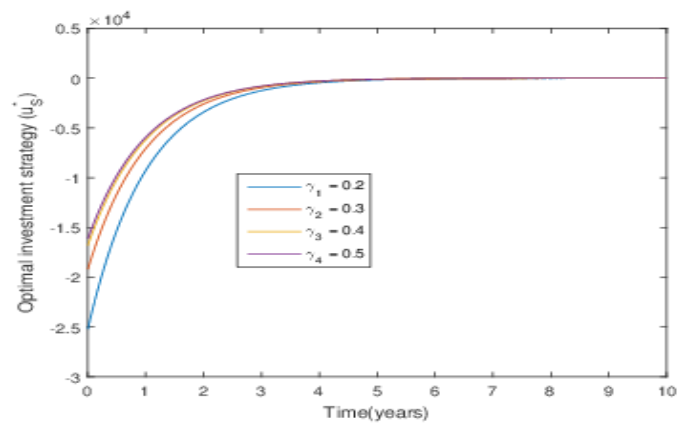

FIGURE VI. IMPACT OF RISK-AVERSION $\gamma$ ON STOCK

\section{CONCLUSION}

This paper devoted to examining the optimal investment strategies for a DC pension fund in the stochastic environment. The pension fund is a risk-averse and uses the power utility function. The pension fund manager is allowed to invest capital in the bank account, stock, and real estate securities and aim to maximize her utility from terminal wealth in a complete market setting where the interest rate dynamics evolve according to the Hull-White interest model. We derived the optimal investment policies and the value function for our problem. The dynamic programming approach was employed to obtain closed-form expressions for the optimal investment strategies under the power utility function. Results show that the price of risk assets and optimal investment strategies increases with time.

\section{ACKNOWLEDGEMENT}

This study is supported by the National Natural Science Foundation of China under Project No: 11071029.

\section{REFERENCES}

[1] Devolder, P., Janssen, J., and Manca, R, “On certain integrals of Optimal Control of a Defined Contribution Pension Scheme. John Wiley and Sons, Inc., 2013.

[2] Boulier, J.-F., Huang, S., and Taillard, G., “Optimal management under stochastic interest rates: the case of a protected defined contribution pension fund," Insurance: Mathematics and Economics, vol. 28, no. 2, pp. 173 - 189, 2001.

[3] Ma, Q.-P., "On optimal pension management in a stochastic framework with exponential utility,” Insurance: Mathematics and Economics, vol. 49, no. 1, pp. $61-69,2011$.

[4] Battocchio, P. and Menoncin, F., "Optimal pension management in a stochastic framework,” Insurance: Mathematics and Economics, vol. 34, no. 1 , pp. $79-95,2004$. 
[5] Josa-Fombellida, R. and Rincn-Zapatero, J. P., "Optimal asset allocation for aggregated defined benefit pension funds with stochastic interest rates," European Journal of Operational Research, vol. 201, no. 1, pp. 211 - 221, 2010.

[6] Deelstra, G., Grasselli, M., and Koehl, P.-F., "Optimal investment strategies in the presence of a minimum guarantee," Insurance: Mathematics and Economics, vol. 33, no. 1, pp. 189 - 207, 2003.

[7] Wei Han, N. and Wei Hung, M., "Optimal asset allocation for dc pension plans under inflation,” Insurance: Mathematics and Economics, vol. 51, no. 1, pp. 172 - 181, 2012.

[8] Zhang, C. and Rong, X., "Optimal investment strategies for dc pension with stochastic salary under the affine interest rate model," Discrete Dynamics in Nature and Society, vol. 2013, 2013.

[9] Zhang, C.-b., Rong, X.-m., Zhao, h., and Hou, R.-j.. “Optimal investment for the defined-contribution pension with stochastic salary under a cev model,” Applied Mathematics-A Journal of Chinese Universities, vol. 28, no. 2, pp. 187-203, Jun 2013.

[10] Guan, G. and Liang, Z., "Optimal management of dc pension plan in a stochastic interest rate and stochastic volatility framework," Insurance: Mathematics and Economics, vol. 57, pp. 58-66, 2014.

[11] Vigna, E. and Haberman, S., "Optimal investment strategy for defined contribution pension schemes," Insurance: Mathematics and Economics, vol. 28, no. 2, pp. 233 - 262, 2001.

[12] Haberman, S. and Vigna, E., "Optimal investment strategies and risk measures in defined contribution pension schemes," Insurance: Mathematics and Economics, vol. 31, no. 1, pp. 35 - 69.

[13] Gao, J., "Optimal portfolios for dc pension plans under a cev model," Insurance: Mathematics and Economics, vol. 44, no. 3, pp. 479 - 490, 2009.

[14] Vigna, E., "On efficiency of mean-variance based portfolio selection in defined contribution pension schemes," Quantitative finance, vol. 14, no. 2, pp. 237-258, 2014.

[15] Musiela, M. and Rutkowski, M., "Martingale methods in financial modeling, (2005)." 